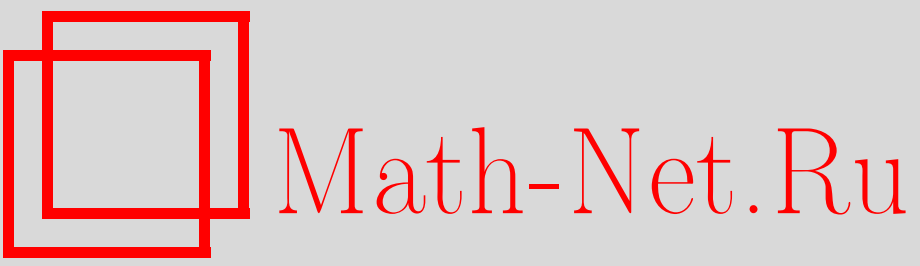

В. В. Глаголев, Л. В. Глаголев, А. А. Маркин, Взаимодействие связанных пластин в однородном температурном поле, Итоги науки и техн. Сер. Соврем. мат. и ее прил. Темат. обз., 2020, том 186, 32-37

DOI: https://doi.org/10.36535/0233-6723-2020-186-32-37

Использование Общероссийского математического портала Math-Net.Ru подразумевает, что вы прочитали и согласны с пользовательским соглашением

http://www.mathnet.ru/rus/agreement

Параметры загрузки:

IP: 54.92 .164 .108

26 апреля 2023 г., 16:03:35 


\title{
ВЗАИМОДЕЙСТВИЕ СВЯЗАННЫХ ПЛАСТИН В ОДНОРОДНОМ ТЕМПЕРАТУРНОМ ПОЛЕ
}

\author{
(c) 2020 г. $\quad$ В. В. ГЛАГОЛЕВ, Л. В. ГЛАГОЛЕВ, А. А. МАРКИН
}

\begin{abstract}
АннотАция. Рассматривается равновесное термоупругое состояние двух пластин, связанных тонким слоем. Задача сводится к системе шести дифференциальных уравнений второго порядка. Рассмотрены температурные деформации биметаллической пластины, защемленной на одном краю и свободной на другом. В случае нулевой толщины слоя взаимодействия получен закон изменения линии сопряжения и распределение напряжений вдоль этой линии. При однородном состоянии (чистый изгиб) кривизна линии сопряжения становится постоянной. Для нулевого коэффициента Пуассона получена известная зависимость кривизны от температуры.
\end{abstract}

Ключевъе слова: биметаллическая пластина, однородное температурное воздействие.

\section{INTERACTION OF BONDED PLATES IN A UNIFORM TEMPERATURE FIELD}

\author{
(c) 2020 V. V. GLAGOlEV, L. V. GLAGOLEV, A. A. MARKIN
}

\begin{abstract}
An equilibrium thermoelastic state of two plates connected by a thin layer is examined. The problem is reduced to a system of six second-order differential equations. The temperature deformations of a bimetallic plate clamped at one edge and free at the other are considered. In the case of zero thickness of the interaction layer, the law of change of the conjugation line and the distribution of stresses along this line are obtained. In a uniform state (pure bend), the curvature of the mating line becomes constant. For zero Poisson ratio, the temperature dependence of the curvature is obtained.
\end{abstract}

Keywords and phrases: bimetallic plate, uniform temperature effect.

AMS Subject Classification: 74K20, 74F05, 34A30

1. Постановка задачи. Рассматривается биметаллическая пластина, схема которой представлена на рис. 1 . Пластины 1 и 2 с толщинами $h_{1}$ и $h_{2}$ связаны слоем 3 толщиной $\delta_{0}$.

Материал слоя 3 может быть клеевой подложкой либо сплавом тел 1 и 2 . Предполагается, что нагрев биметаллической пластины осуществлен на величину $\Delta T$ от начального состояния и распределение температуры однородно. Левый торец пластины жестко закреплен от перемещений, а вся остальная поверхность свободна от внешней нагрузки.

Работа выполнена при поддержке Российского фонда фундаментальных исследований (проекты № 18-31-20053 и № 19-41-710001p_a). 


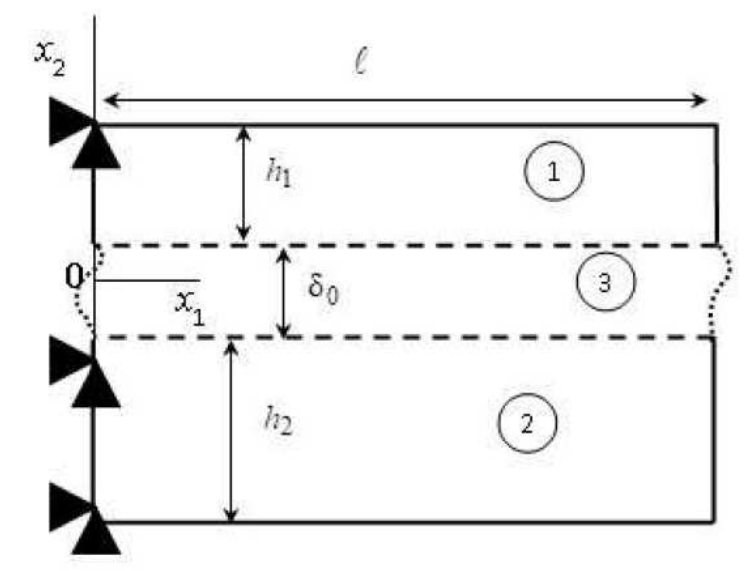

Рис. 1. Биметаллическая пластина с адгезионным слоем

На основе концепции «слоя взаимодействия», развитой в работах [5,6], в статье [1] получена система дифференциальных уравнений следующего вида:

$$
\begin{array}{lll}
\frac{d M_{11}^{(1)}}{d x_{1}}-Q_{12}^{(1)}=0, & \frac{d M_{11}^{(2)}}{d x_{1}}-Q_{12}^{(2)}=0, & \frac{d Q_{11}^{(1)}}{d x_{1}}+\frac{1}{2} \delta_{0} \frac{d \bar{\sigma}_{11}}{d x_{1}}=\bar{\sigma}_{21}, \\
\frac{d Q_{12}^{(1)}}{d x_{1}}+\frac{1}{2} \delta_{0} \frac{d \bar{\sigma}_{21}}{d x_{1}}=\bar{\sigma}_{22}, & \frac{d Q_{12}^{(2)}}{d x_{1}}+\frac{1}{2} \delta_{0} \frac{d \bar{\sigma}_{21}}{d x_{1}}=-\bar{\sigma}_{22}, & \frac{d Q_{11}^{(2)}}{d x_{1}}+\frac{1}{2} \delta_{0} \frac{d \bar{\sigma}_{11}}{d x_{1}}=-\bar{\sigma}_{21},
\end{array}
$$

где $Q_{1 k}^{(1)}\left(x_{1}\right), Q_{1 k}^{(2)}\left(x_{1}\right)$ - обобщенные силы [2]; $M_{11}^{(1)}\left(x_{1}\right), M_{11}^{(2)}\left(x_{1}\right)$ - обобщенные моменты [2]; $\bar{\sigma}_{m k}-$ средние напряжения в слое $[5,6] ; m, k=1,2$. Поле перемещений в телах 1 и 2 определялось следующим образом: $u_{1}^{(1)}=u_{1}^{+}\left(x_{1}\right)-\varphi_{1}\left(x_{1}\right)\left(x_{2}-\delta_{0} / 2\right), u_{2}^{(1)}=u_{2}^{+}\left(x_{1}\right) u_{1}^{(2)}=u_{1}^{-}\left(x_{1}\right)-\varphi_{2}\left(x_{1}\right)\left(x_{2}+\delta_{0} / 2\right)$, $u_{2}^{(2)}=u_{2}^{-}\left(x_{1}\right)$, где параметры $\varphi_{k}$ имеют геометрический смысл малых углов поворота материальных нормалей к плоскостям $x_{2}= \pm \delta_{0} / 2$ в телах 1 и $2 ; u_{k}^{ \pm}$- компоненты вектора перемещения границ слоя.

Согласно распределению поля перемещений отличные от нуля деформации в теле 1 будут определяться в виде

$$
\varepsilon_{11}^{(1)}=\frac{d u_{1}^{+}}{d x_{1}}-\varphi_{1}^{\prime}\left(x_{1}\right)\left(x_{2}-\delta_{0} / 2\right), \quad \varepsilon_{12}^{(1)}=\varepsilon_{21}^{(1)}=\frac{1}{2}\left(\frac{d u_{2}^{+}}{d x_{1}}-\varphi_{1}\left(x_{1}\right)\right),
$$

а в теле 2

$$
\varepsilon_{11}^{(2)}=\frac{d u_{1}^{-}}{d x_{1}}-\varphi_{2}^{\prime}\left(x_{1}\right)\left(x_{2}+\delta_{0} / 2\right), \quad \varepsilon_{12}^{(2)}=\varepsilon_{21}^{(2)}=\frac{1}{2}\left(\frac{d u_{2}^{-}}{d x_{1}}-\varphi_{2}\left(x_{1}\right)\right) .
$$

Выражения (2), (3), как и соотношения теории Тимошенко [2], учитывают сдвиговые деформации и повороты нормалей в телах 1 и 2.

Определяющие соотношения для тел 1 и 2 принимаем в форме Дюгамеля-Неймана [3]:

$$
\sigma_{i j}=\frac{E_{k}}{1+\nu_{k}}\left(\varepsilon_{i j}+\frac{\nu_{k}}{1-2 \nu_{k}} \varepsilon \delta_{i j}\right)-\frac{E_{k}}{1-2 \nu_{k}} \alpha_{k} \Delta T \delta_{i j}
$$

где $E_{k}, v_{k}$ - модуль упругости и коэффициент Пуассона $k$-го тела; $\alpha_{k}-$ коэффициент температурного расширения; $\varepsilon=\varepsilon_{11}+\varepsilon_{22}+\varepsilon_{33}$ - объемное расширение; $\delta_{i j}$ - символ Кронекера; $i, j=1,2,3$.

Рассмотрен вариант сопряжения уравнений системы (1) условиями: $u_{k}=u_{k}^{+}=u_{k}^{-}$. В этом случае положим в $(1) \delta_{0}=0$. При этом средние напряжения слоя будут совпадать со значениями 
проекций векторов напряжений взаимодействующих пластин и определяться из связи с обобщенными силами:

$$
\frac{d Q_{11}^{(1)}}{d x_{1}}=\bar{\sigma}_{21}, \quad \frac{d Q_{12}^{(1)}}{d x_{1}}=\bar{\sigma}_{22}
$$

Из (1) получим условия равновесия пластин в виде системы четырех дифференциальных уравнений:

$$
\frac{d M_{11}^{(k)}}{d x_{1}}-Q_{12}^{(k)}=0, \quad Q_{1 k}^{(1)}+Q_{1 k}^{(2)}=0
$$

Обобщенные силы и моменты с учетом выражений (2) -(4) определяются по формулам:

$$
\begin{gathered}
Q_{11}^{(k)}=D_{k}\left(h_{k} \frac{d u_{1}}{d x_{1}} \mp \frac{h_{k}^{2}}{2} \varphi_{k}^{\prime}\right)-h_{k} U_{k} \alpha_{k} \Delta T, \quad Q_{12}^{(k)}=L_{k} h_{k}\left(\frac{d u_{2}}{d x_{1}}-\varphi_{k}\right), \\
M_{11}^{(k)}= \pm D_{k}\left(\frac{h_{k}^{2}}{2} \frac{d u_{1}}{d x_{1}} \mp \frac{h_{k}^{3}}{3} \varphi_{k}^{\prime}\right) \mp \frac{h_{k}^{2} U_{k} \alpha_{k}}{2} \Delta T
\end{gathered}
$$

где для символов $\mp, \pm$ верхний знак соответствует $k=1$,

$$
D_{k}=\frac{E_{k}\left(1-\nu_{k}\right)}{\left(1+\nu_{k}\right)\left(1-2 \nu_{k}\right)}, \quad U_{k}=\frac{E_{k}}{1-2 \nu_{k}}, \quad L_{k}=\frac{E_{k}}{2\left(1+\nu_{k}\right)} .
$$

Основными неизвестными в этом случае будут четыре функции $u_{k}, \varphi_{k}$. Решение системы (6) с граничными условиями

получено в следующем виде:

$$
\left.u_{k}^{ \pm}\left(x_{1}\right)\right|_{x_{1}=0}=0,\left.\quad \varphi_{k}\left(x_{1}\right)\right|_{x_{1}=0}=0,\left.\quad M_{11}^{(k)}\right|_{x_{1}=\ell}=0,\left.\quad Q_{1 k}^{(2)}\right|_{x_{1}=\ell}=0
$$

$$
\begin{aligned}
u_{1} & =(c-d) s_{1} C_{2} x_{1}-2 C_{1} \operatorname{sh}\left(s_{3} x_{1}\right)(c-\mu d)+t x_{1}, \\
u_{2} & =\frac{1}{2}(a+b) s_{1} C_{2} x_{1}^{2}-\frac{2}{s_{3}}(a+\mu b) C_{1}\left(\operatorname{ch}\left(s_{3} x_{1}\right)-1\right), \\
\varphi_{1} & =C_{2} s_{1} x_{1}-2 C_{1} \operatorname{sh}\left(s_{3} x_{1}\right), \quad \varphi_{2}=C_{2} s_{1} x_{1}-2 \mu C_{1} \operatorname{sh}\left(s_{3} x_{1}\right),
\end{aligned}
$$

где

$$
\begin{gathered}
C_{1}=\frac{\frac{(c-d) \Delta T}{4}\left(\frac{U_{1} \alpha_{1}}{D_{1}}-\frac{U_{2} \alpha_{2}}{D_{2}}\right)+\frac{\Delta T}{6}\left(\frac{U_{1} \alpha_{1} h_{2}}{D_{1}}+\frac{U_{2} \alpha_{2} h_{1}}{D_{2}}\right)-\frac{t}{6}\left(h_{1}+h_{2}\right)}{s_{3} \operatorname{ch}\left(s_{3} \ell\right)\left(\frac{(2 c-d)}{6}\left(h_{1}+\mu h_{2}\right)+\frac{2 h_{1} h_{2}}{9}(1-\mu)+\frac{\left(h_{1}+h_{2}\right)}{3}(\mu d-c)\right),} \\
C_{2}=\frac{\frac{U_{2} \alpha_{2} \Delta T}{2 D_{2}}\left(\frac{2 h_{1}}{3}-c+\mu d\right)+\frac{U_{1} \alpha_{1} \Delta T}{2 D_{1}}\left(\frac{2 \mu h_{2}}{3}+c-\mu d\right)-\frac{2 t}{6}\left(h_{1}+\mu h_{2}\right)}{s_{1}\left(\frac{(2 c-d)}{6}\left(h_{1}+\mu h_{2}\right)+\frac{2 h_{1} h_{2}}{9}(1-\mu)+\frac{\left(h_{1}+h_{2}\right)}{3}(\mu d-c)\right)}, \\
c=\frac{D_{k}=\frac{E_{k}\left(1-\nu_{k}\right)}{\left(1+\nu_{k}\right)\left(1-2 \nu_{k}\right)}, \quad L_{k}=\frac{E_{k}}{2\left(1+\nu_{k}\right)}, \quad U_{k}=\frac{E_{k}}{1-2 \nu_{k}},}{2\left(D_{1} h_{1}+D_{2} h_{2}\right)}, \quad d=\frac{D_{2} h_{2}^{2}}{2\left(D_{1} h_{1}+D_{2} h_{2}\right)}, \quad t=\frac{\left(h_{1} U_{1} \alpha_{1}+h_{2} U_{2} \alpha_{2}\right) \Delta T}{\left(D_{1} h_{1}+D_{2} h_{2}\right)}, \\
a=\frac{L_{1} h_{1}}{\left(L_{1} h_{1}+L_{2} h_{2}\right)}, \quad b=\frac{L_{2} h_{2}}{\left(L_{1} h_{1}+L_{2} h_{2}\right)}, \\
z=D_{1}\left(\frac{h_{1}^{2} c}{2}-\frac{h_{1}^{3}}{3}\right), \quad q=D_{1} \frac{h_{1}^{2} d}{2}, \quad y=L_{1} h_{1}(a-1), \\
L_{1} h_{1} b, \quad z_{1}=-D_{2} \frac{h_{2}^{2} c}{2}, \quad q_{1}=D_{2}\left(\frac{h_{2}^{3}}{3}-\frac{h_{2}^{2} d}{2}\right), \quad y_{1}=L_{2} h_{2} a, \quad p_{1}=L_{2} h_{2}(b-1),
\end{gathered}
$$




$$
\begin{gathered}
s=\frac{q_{1}}{z_{1}} r+\frac{y_{1}}{z_{1}}, \quad s_{1}=\frac{q_{1}}{z_{1}} r_{1}+\frac{p_{1}}{z_{1}}, \quad r=\frac{y / z-y_{1} / z_{1}}{q_{1} / z_{1}-q / z}, \\
r_{1}=\frac{p / z-p_{1} / z_{1}}{q_{1} / z_{1}-q / z}, \quad \mu=\frac{r}{s}, \quad s_{3}=\sqrt{s+\mu s_{1}}
\end{gathered}
$$

- постоянные, зависящие от геометрических, механических свойств пластин и изменения температуры $\Delta T$.

Приведем вариант решения для однородного распределения по длине пластины характеристик НДС. В этом случае $\frac{d M_{11}^{(k)}}{d x_{1}}=0$. Тогда из (6) следует, что $Q_{12}^{(k)}=0$. Поэтому сдвиговые деформации отсутствуют и на основании выражений (2), (3) углы поворотов совпадают:

$$
\varepsilon_{12}^{(k)}=0, \quad \varphi_{k}=\frac{d u_{2}}{d x_{1}}=\varphi_{0} .
$$

С учетом граничных условий из системы (5) получаем первые интегралы:

$$
M_{11}^{(1)}+M_{11}^{(2)}=0, \quad Q_{11}^{(1)}+Q_{11}^{(2)}=0,
$$

где основными неизвестными являются компонента перемещения поверхности сопряжения материалов $u_{1}$ и функция $\varphi_{0}$.

Из системы (8) получено распределение искомых функций при отсутствии в телах 1 и 2 сдвиговых деформаций ввиду однородности решения:

$$
\begin{gathered}
\varphi_{0}=\frac{6 h_{1} h_{2}\left(h_{1}+h_{2}\right)\left(D_{1} U_{2} \alpha_{2}-D_{2} U_{1} \alpha_{1}\right) \Delta T x_{1}}{4\left(D_{1} h_{1}+D_{2} h_{2}\right)\left(D_{1} h_{1}^{3}+D_{2} h_{2}^{3}\right)-3\left(D_{1} h_{1}^{2}-D_{2} h_{2}^{2}\right)^{2}}, \\
u_{2}=\frac{3 h_{1} h_{2}\left(h_{1}+h_{2}\right)\left(D_{1} U_{2} \alpha_{2}-D_{2} U_{1} \alpha_{1}\right) \Delta T x_{1}^{2}}{4\left(D_{1} h_{1}+D_{2} h_{2}\right)\left(D_{1} h_{1}^{3}+D_{2} h_{2}^{3}\right)-3\left(D_{1} h_{1}^{2}-D_{2} h_{2}^{2}\right)^{2}}, \\
u_{1}=\frac{\left(D_{1} U_{1} h_{1}^{4} \alpha_{1}+D_{2} U_{2} h_{2}^{4} \alpha_{2}+h_{1} h_{2}\left(D_{1} U_{2} \alpha_{2}\left(4 h_{1}^{2}+3 h_{1} h_{2}\right)+D_{2} U_{1} \alpha_{1} t\left(4 h_{2}^{2}+3 h_{1} h_{2}\right)\right)\right) \Delta T x_{1}}{4\left(D_{1} h_{1}+D_{2} h_{2}\right)\left(D_{1} h_{1}^{3}+D_{2} h_{2}^{3}\right)-3\left(D_{1} h_{1}^{2}-D_{2} h_{2}^{2}\right)^{2}} .
\end{gathered}
$$

Найдена кривизна линии сопряжения материалов:

$$
\varphi_{0}^{\prime}=\frac{6 h_{1} h_{2}\left(h_{1}+h_{2}\right)\left(D_{1} U_{2} \alpha_{2}-D_{2} U_{1} \alpha_{1}\right) \Delta T}{4\left(D_{1} h_{1}+D_{2} h_{2}\right)\left(D_{1} h_{1}^{3}+D_{2} h_{2}^{3}\right)-3\left(D_{1} h_{1}^{2}-D_{2} h_{2}^{2}\right)^{2}} .
$$

В отличие от формулы статьи [4] последнее выражение определяет кривизну не нейтральной, а граничной линии. Эта формула учитывает возможность искривления при $\alpha_{1}=\alpha_{2}$, но $D_{1} U_{2} \neq$ $D_{2} U_{1}$. Если положить в последнем соотношении $\nu_{1}=\nu_{2}=0$, то получим формулу статьи [4]:

$$
\varphi_{0}^{\prime}=\frac{6 h_{1} h_{2}\left(h_{1}+h_{2}\right) E_{1} E_{2}\left(\alpha_{2}-\alpha_{1}\right) \Delta T}{4\left(E_{1} h_{1}+E_{2} h_{2}\right)\left(E_{1} h_{1}^{3}+E_{2} h_{2}^{3}\right)-3\left(E_{1} h_{1}^{2}-E_{2} h_{2}^{2}\right)^{2}} .
$$

Из решения (7) найден закон изменения кривизны линии сопряжения пластин в общем случае свободного правого торца:

$$
\frac{d^{2} u_{2}}{d x_{1}^{2}}=(a+b) s_{1} C_{2}-2(a+\mu b) C_{1} s_{3} \operatorname{ch}\left(s_{3} x_{1}\right)
$$

и распределения напряжений взаимодействия пластин:

$$
\begin{gathered}
\bar{\sigma}_{21}=D_{1} h_{1}\left(\left(c-d-\frac{h_{1}}{2}\right) s_{1} C_{2}-2 s_{3} C_{1} \operatorname{ch}\left(s_{3} x_{1}\right)\left(c-\mu d+\frac{h_{1}}{2}\right)+t\right), \\
\bar{\sigma}_{22}=L_{1} h_{1}\left((a+b-1) s_{1} C_{2} x_{1}-2(a+\mu b-1) C_{1} \operatorname{sh}\left(s_{3} x_{1}\right)\right) .
\end{gathered}
$$

2. Результаты расчетов. Проведены расчеты для биметаллической пластины со следующими механическими характеристиками тела $1: E_{1}=7 \cdot 10^{10}$ Па, $\nu_{1}=0,35, \alpha_{1}=22,8 \cdot 10^{-6}$, соответствующими алюминию и материала тела $2: E_{2}=2 \cdot 10^{11}$ Па, $\nu_{2}=0,3, \alpha_{2}=11,3 \cdot 10^{-6}$, соответствующие стали. Толщина тела 1 выбиралась равной $h_{1}=0,01 \mathrm{~m}$, а тела $2-h_{2}=0,007$ м. Длина биметаллической пластины $-\ell=0,3 \mathrm{м}$, а изменение ее температуры $-\Delta T=50^{\circ}$. На рис. 2 показан закон изменения кривизны (9). 


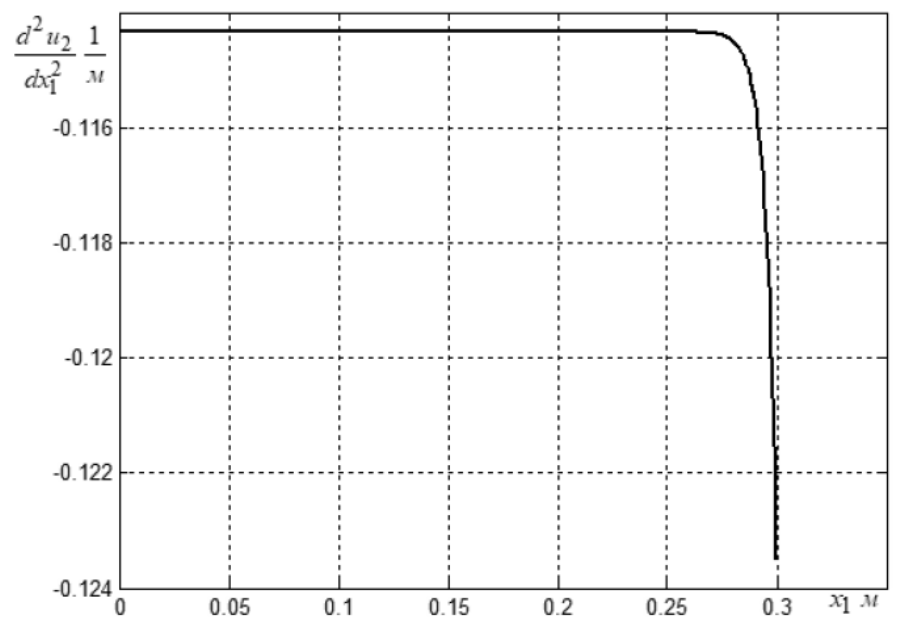

Рис. 2. Распределение кривизны линии сопряжения биметаллической пластины

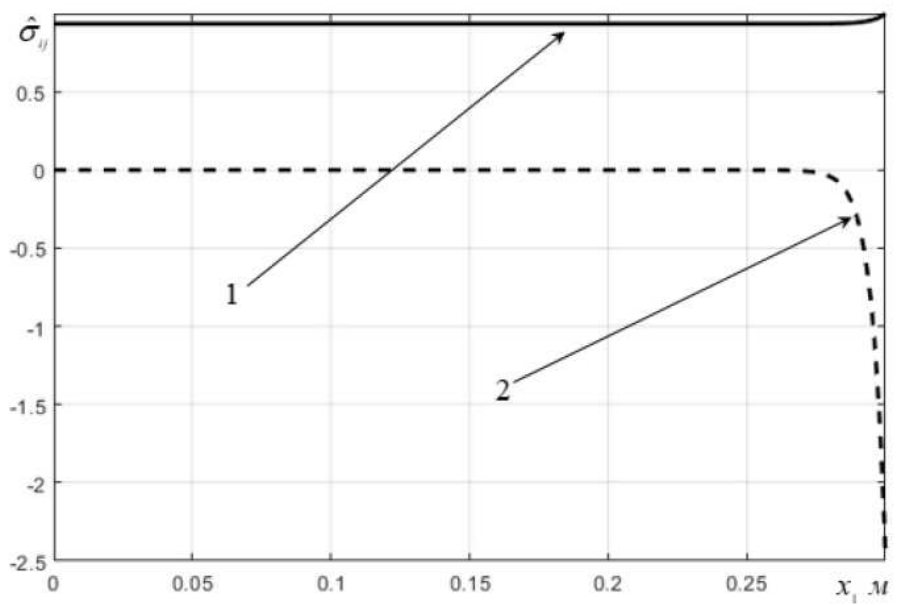

Рис. 3. Распределение напряжений взаимодействия

Из рис. 2 видно, что в отличие от «чистого» изгиба кривизна не является постоянной и меняет свое значение в концевой области свободного торца пластины. Данный результат показывает, что влияние сдвиговых деформаций в сопрягаемых пластинах имеет характер краевого эффекта.

На рис. 3 приведен закон распределения безразмерных напряжений взаимодействия пластин. Напряжения (10) и (11) отнесены к значению напряжения (10) на правом краю линии сопряжения: $\hat{\sigma}_{21}=\bar{\sigma}_{21} /\left.\bar{\sigma}_{21}\right|_{x_{1}=\ell}, \hat{\sigma}_{22}=\bar{\sigma}_{22} /\left.\bar{\sigma}_{21}\right|_{x_{1}=\ell}$.

График 1 на рис. 3 определяет напряжение $\hat{\sigma}_{21}$, а график $2-\hat{\sigma}_{22}$. Из приведенных расчетов видно, что хотя напряжения $\hat{\sigma}_{22}$ в зоне краевого эффекта значительно превосходят сдвиговые, но являются сжимающими. В этом случае возможное разрушение будет формироваться за счет сдвиговых адгезионных взаимодействий пластин.

\section{СПИСОК ЛИТЕРАТУРЫ}

1. Глаголев В. В., Маркин А. А., Пашинов С. В. Биметаллическая пластина в однородном температурном поле// Мех. композ. мат. констр. - 2017. - 23, № 3. - С. 331-343.

2. Тимошенко С. П., Войновский-Кригер С. Пластины и оболочки. - М.: Физматгиз, 1963. 
3. Хан X. Теория упругости: Основы линейной теории ее применения. - М.: Мир, 1988.

4. Clyne T. W. Residual stresses in surface coatings and their effects on interfacial debonding// Key Eng. Mat. - 1996. - 116-117. - P. 307-330.

5. Glagolev V. V., Glagolev L. V., Markin A. A. Stress-strain state of elastoplastic bodies with crack// Acta Mech. Solid. Sinica. — 2015. - 28, № 4. - P. 375-383.

6. Glagolev V. V., Markin A. A. Fracture models for solid bodies, based on a linear scale parameter// Int. J. Solids Struct. — 2019. — 158. - P. 141-149.

Глаголев Вадим Вадимович

Тульский государственный университет

E-mail: vadim@tsu.tula.ru

Глаголев Леонид Вадимович

Тульский государственный университет

E-mail: len4ic92@gmail.com

Маркин Алексей Александрович

Тульский государственный университет

E-mail: markin-nikram@yandex.ru 